Citation Information: B. Duffy, J. Thiyagalingam, S. Walton, D. J. Smith,

A. Trefethen, J. C. Kirkman-Brown, E. A. Gaffney and. M. Chen, "Glyph-based

\title{
Glyph-Based Video Visualization for Semen Analysis
}

\author{
Brian Duffy, Member, IEEE, Jeyarajan Thiyagalingam, Simon Walton, David J. Smith, \\ Anne Trefethen, Jackson C. Kirkman-Brown, Eamonn A. Gaffney and Min Chen, Member, IEEE
}

\begin{abstract}
The existing efforts in computer assisted semen analysis have been focused on high speed imaging and automated image analysis of sperm motility. This results in a large amount of data, and it is extremely challenging for both clinical scientists and researchers to interpret, compare and correlate the multidimensional and time-varying measurements captured from video data. In this work, we use glyphs to encode a collection of numerical measurements taken at a regular interval and to summarize spatio-temporal motion characteristics using static visual representations. The design of the glyphs addresses the needs for (a) encoding some 20 variables using separable visual channels, (b) supporting scientific observation of the interrelationships between different measurements and comparison between different sperm cells and their flagella, and (c) facilitating the learning of the encoding scheme by making use of appropriate visual abstractions and metaphors. As a case study, we focus this work on video visualization for computer-aided semen analysis, which has a broad impact on both biological sciences and medical healthcare. We demonstrate that glyph-based visualization can serve as a means of external memorization of video data as well as an overview of a large set of spatiotemporal measurements. It enables domain scientists to make scientific observation in a cost-effective manner by reducing the burden of viewing videos repeatedly, while providing them with a new visual representation for conveying semen statistics.
\end{abstract}

Index Terms-Video visualization, glyph visualization, multivariate data, semen analysis, flagellum locomotion.

\section{INTRODUCTION}

Over the past decade, researchers have demonstrated the usefulness of video visualization through applications such as surveillance [23], [16], sports analysis [58] and entertainment [24], [49]. In biology today, videos are used extensively to capture motile cells, many of which exhibit a tail-like appendage called a flagellum. For example, biomathematicians use video data to reverse-engineer the mechanics of naturally occurring phenomenona such as a cell's flagellum. The most commonly encountered flagella are in the sperm cells of mammals. The motion of a swimming sperm, which is caused by the complex movement of the flagellum, is difficult to characterize using only elementary measurements such as velocity, because it varies in time, and rarely follows a straight line. As shown in Fig. 1, the existing visual representations for such a motion typically depict only the motion path of the cell head, or a sequence of geometries of the flagellum. Whilst Fig. 1(a)

- B. Duffy is with the Oxford Centre for Collaborative Applied Mathematics (OCCAM), University of Oxford. E-mail: bmduffy@gmail.com

- J. Thiyagalingam is with MathWorks, UK. Email: jeyan@bcs.org

- S. Walton, A. Trefethen and M. Chen are with the Oxford eResearch Center (OeRC), University of Oxford. Email: \{simon.walton, anne.trefethen,min.chen $\} @ o e r c . o x . a c . u k$

- D. J. Smith is with the School of Mathematics, University of Birmingham.Email: d.j.smith.2@bham.ac.uk

- E. A. Gaffney is with the Centre for Mathematical Biology (CMB), University of Oxford. Email: gaffney@maths.ox.ac.uk

- J. C. Kirkman-Brown is with The Centre for Human Reproductive Science at the Birmingham Women's Hospital and the School of Clinical and Experimental Medicine, University of Birmingham. Email: j.kirkmanbrown@bham.ac.uk conveys a limited amount of information, it is non-trivial to discern meaningful motion characteristics from those in Fig. 1(b,c). One challenge is to devise an effective visual representation that can summarize the motion of many cells in a video, while depicting a collection of measurable motion characteristics.

Computer-Assisted Semen Analysis (CASA) is a collection of techniques for measuring and analyzing a semen sample captured using a camera or a digitizing tablet through a microscope [45], [2], [70], [46]. Typical measurements include sperm count and up to nine different motility parameters [70]. In recent years, scientists have proposed a number of new parameters for measuring flagellar motion and cell shape morphology (e.g., [20], [62]). This work was inspired by the scientific endeavor to find better prognostic measurements. It was motivated partly by the need for scientists to make observations about the relationships among many different measurements, and partly by the challenge of summarizing cell and flagellum motion, which has not been tackled previously in video visualization.

In order to address the need for depicting a large set of parameters while maintaining spatiotemporal context associated with individual sperm, we propose the use of purposely-designed glyphs to encode the multivariate data space. As shown in Fig. 2, this approach provides domain scientists with a summarization of the motions in the video, and allows them to observe and compare some 20 motility measurements. It offers a spatiotemporal summary of the video and an overview of a large collection of measurements. Conventional visual representations, such as time series plots and parallel coordinates, have difficul- 


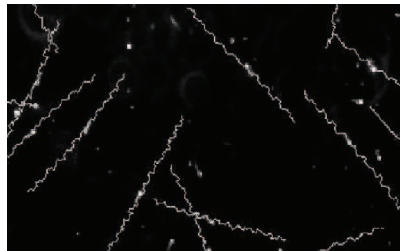

(a) head motion trajectory

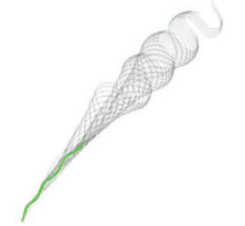

(b) flagellum tracking

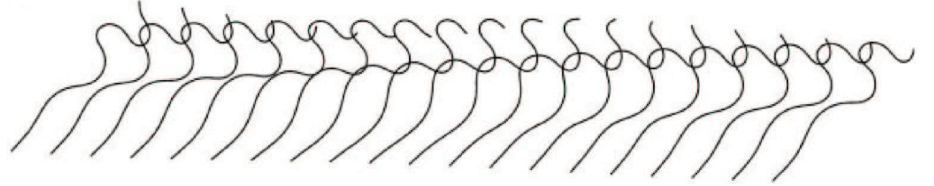

(c) temporal changes of flagellar shapes

Fig. 1: Existing visualizations of sperm depict a limited number of motion attributes. Images are from [61].

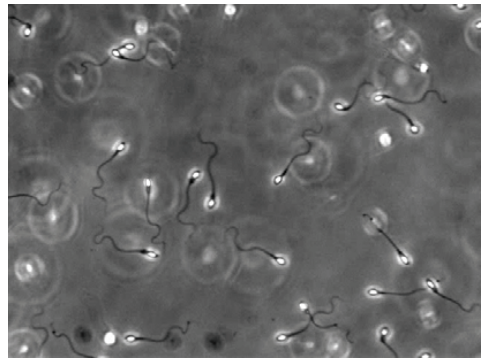

(a) single video frame

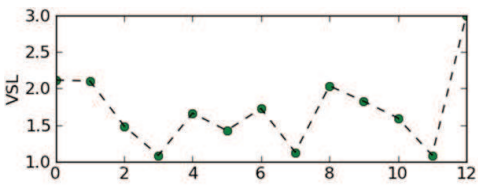

(b) time series plot

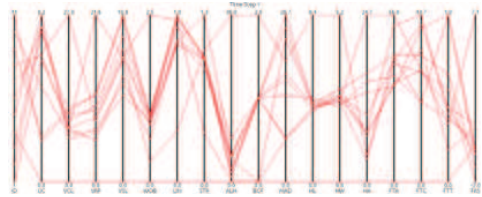

(c) parallel coordinates plot

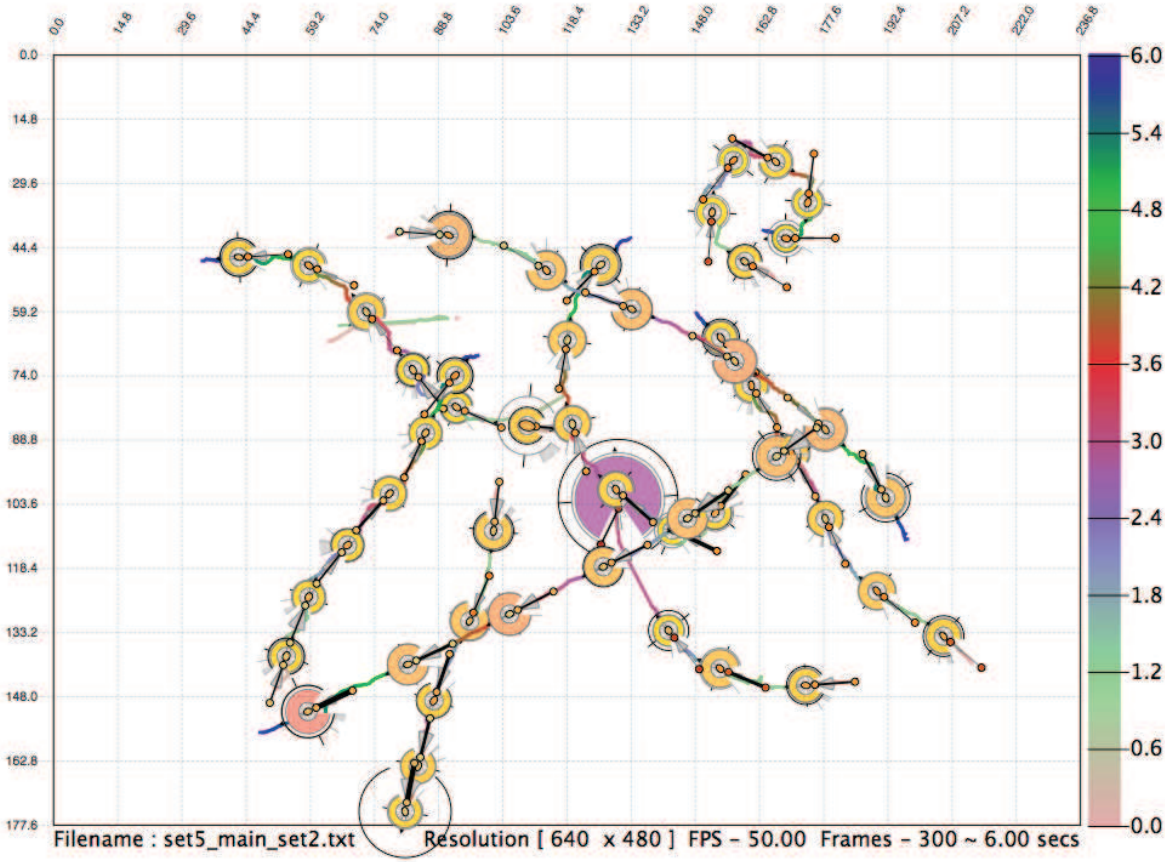

(d) glyph-based video summarization

Fig. 2: (a) Watching video is intuitive, but it is ineffective and inefficient for observing and comparing the motility measurements of different cells. (b) A time series plot is useful for observing one measure each time, but cannot convey spatial information and context effectively. (c) A parallel coordinates plot can be used to observe relationships between different measurements, but cannot convey temporal variations. (d) a glyph-based visualization can summarize a video by conveying multiple measurements with both spatial and temporal contexts.

ties to convey such an overview. Most existing work on glyph-based visualization typically deals with 3-5 attribute dimensions, with perhaps the highest dimensionality in glyph-based visualization for DTI datasets [15], [35]. It is therefore not a trivial design problem to encode a data space with some 20 dimensions. While we recognize an advantageous fact that many scientists, engineers and other professionals are accustomed to schematic representations featuring abstract or metaphoric visual encoding such as icons, it is still desirable to facilitate effective learning, memorizing and using the encoding scheme by domain specialists. Our contributions are as follows:

- We have developed a novel visualization method for the summarization and depiction of sperm and flagellum motion captured on videos;

- We have stretched the capability of glyph-based visualization to encode a high-dimensional data space with some 20 explicit and 3 implicit parameters, while making use of appropriate abstractions and metaphors to facilitate the learning of the encoding scheme;
- We have provided scientists with a tool for making observations of multivariate measurements, and comparing different cells and their flagella in the parameter space with both spatial and temporal contexts.

The remainder of this paper is organized as follows. In Section 2, we give a brief overview of current state-ofthe-art in video and glyph-based visualization. In Section 3 we provide the application background of this work and describe our analysis of the visualization tasks. In Section 4 we describe the data space that encapsulates the WHOrecommended measures and additional measures proposed by biomathematicians. In addition, we outline the process for obtaining various measurements from videos and discuss the need to consider uncertainty in visualization. In Section 6, we describe the design principles followed by this work and the process of attaining the effective glyph design for this application. In Section 7, we present and discuss the results of visualization, together with a brief description of our implementation. We report the evaluation by domain specialists in Section 8 , and offer our concluding 
remarks in Section 9.

\section{Related Work}

\subsection{Video Visualization}

Video visualization is a collection of graphics and visualization techniques concerned with the creation of a new visual representation from an input video to reveal important features and events in the video. Meaningful information is extracted from videos and conveyed to users in summary or abstract representations. Video visualization assists users in intelligent reasoning by summarizing video content, removing or reducing the burden of viewing videos. This aim justifies deflection from creation of realistic imagery and instead advocates simplifications, abstraction and embellishments if they improve understanding.

Image-based techniques segment videos into frames, shots and scenes [50]. Keyframes detection is crucial in these applications [65]. There are three different approaches to shortening the time of watching videos: video navigation techniques [41], [53], [59], [17], video montage and video synopsis [32] and video skimming techniques [65], [11].

Abstract techniques summarize attributes of a video (e.g., changes in a scene, changes between frames, motion flow and pixel clusters), depicting these attributes visually to aid understanding of video using a small number of visualizations [14], [10]. Such visualizations may not display objects directly but can convey temporal attributes more effectively than more realistic representations such as keyframes.

The development of the subject has been heavily influenced by many applications in entertainment [24], [49], sports [58], [28], [40], and security [8]. In a recent survey, Borgo et al. [6] proposed a taxonomy to categorize the concepts and techniques in this newly-emerged body of knowledge. They also gave a concise overview of the major advances in automated video analysis, video-based graphics and video visualization.

This work falls into the category of abstract techniques. To our knowledge, it represents the first scientific application of video visualization. The requirement for depicting multivariate measurements about multiple objects poses a problem that is yet to have satisfactory solutions. For example, the solution of [28] featured a few attributes only, and that in [40] omitted most spatial information.

\subsection{Glyph-based Visualization}

Glyph visualization is a form of visual design where data is depicted by a collection of visual objects. Glyphs are often featured in geo-information visualization and schematic diagrams, allowing the patterns of multivariate data to be more readily perceived in a spatial context. Ward [67] first proposed a taxonomy for glyph placement strategies. He later presented a major survey of glyph-based visualization [66], covering aspects of visual mapping and layout, and raising important issues such as bias in mapping and interpretation. Recently, Borgo et al. [7] presented a new survey on glyph-based visualization, featuring historical development of signs, a large collection of design approaches, and several application areas. Bertin [5] provided perhaps the first theoretic study of glyph design in the context of geo-information visualization. Maguire et al. [44] compiled a set of design guidelines based on existing experimental results in perception as well as Bertin's study in the context of workflow visualization.

Glyphs are used extensively in vector field visualization. Post et al. [52] and Laramee et al. [38] compared their uses with other visual representations of flows. Wong et al. [69] presented a glyph layering strategy for visualizing multivariate climate data. Kirby et al. [36] adapted concepts from oil painting to visualise multivariate flow data. Glyphs have been utilized in medical applications to depict multivariate volume data [15], [35], [57], [27]. Further details can be found in the survey reports by Ropinski et al. [56], [55]. Fuchs et al. [19] discussed uses of glyphs in scientific visualization. Crawfis et al. [12] proposed a language for combining data of different frequencies (images, vectors, glyphs, surfaces, etc.) using primitive operators.

Our work built on the above-mentioned works. It was particularly enthused by the glyph-based video visualization in [40] and its successful deployment in practice. We also recognize that our data presents a challenge that existing glyph-based solutions cannot be adapted easily. For example, glyphs in [40] feature many nominal data types, those in vector field visualization encode only a few attribute dimensions, and those in DTI data visualization are not required to encode temporal variations. A new solution is thus necessary.

\section{ApPLICATION BACKGROUND}

\subsection{Computer-aided Semen Analysis (CASA)}

Collectively, Computer-aided Semen Analysis (CASA) refers to all digital techniques for capturing, processing, analyzing and characterizing semen samples. It is an important research area in reproductive sciences. Since the early application of image analysis to semen samples (e.g., [3]), significant advances have been made, especially in estimating sperm concentration and measuring sperm motility. Some techniques have been transferred to commercial systems delivered to hospitals and fertility clinics. In comparison with manual observation methods, CASA provides more objective, and potentially more accurate, means of quantitative measurement.

The development of CASA has been focused on tackling challenging problems in image and video analysis, as accuracy in classifying and tracking cells is often compromised by contaminants of semen such as white blood cells. Manual methods are still used extensively due to accuracy, flexibility or unavailability of costly equipment. The World Health Organization (WHO) currently endorses manual counting as the "gold standard" for measuring sperm concentration [70]. However, manually estimating most other measurements, such as motility and morphology characteristics, is unfeasible. Hence, continuing advancement in CASA is crucial. 
The commercialized technology provides visualization of the trajectories of cells in a video in conjunction with some global statistics through a small set of numerical values. Such abstraction often does not offer an insightful representation of a sample, because in a group of sperm, only a relative few have fertilization potential. In particular, scientists and researchers are usually more interested in a variety of characteristics of individual cells in different spatial and temporal contexts. As the imaging technology and image analysis techniques continue to improve, there is an increasing need for observing and analyzing multivariate parameters of cells.

Since von Leeuwenhoek first used a microscope to observe sperm in 1678 [2], scientists have proposed many measures for characterizing sperm motion. For example, Jecht and Russo [31] reported 5 motion parameters for analysis of sperm videomicrography. Katz et al. [34] considered 7 motion parameters, while Katz and Davis [33] extended the collection to 10 parameters. Subsequently, the WHO laboratory manual [70] defined 9 parameters as the standard measures for sperm motility analysis (see Section 4.1 and Fig. 3 for details). Additionally, researchers in reproductive sciences have been paying increasing attention to flagellar dynamics (e.g., [20]) and shape morphology of sperm (e.g., [62]). Hence, the usability of CASA is no longer just a problem of computer vision. It is equally, if not more, important to enable domain specialists to observe the multifaceted characteristics of sperm in an effective manner. This leads to the necessity for integrating more visualization techniques into CASA.

\subsection{Tasks Analysis}

The work presented in this paper was conducted over a 12 month period, during which tasks analysis was evolved iteratively in conjunction with visual design, prototype development and evaluation in an agile manner [47]. Here we report primarily a snapshot of our tasks analysis captured during the first 3 months. We considered three groups of potential users: (a) biomathematicians studying sperm motility and morphology, (b) biologists specialized in reproductive sciences, and (c) clinical analysts performing semen analysis routinely. During the project, we worked closely with one biomathematician, consulted a team of biologists in several meetings and engaged in detailed discussions with two of them through many emails, and sought advice from two clinical analysts. In addition, the first author was provided with a placement in the Institute of Reproductive Sciences in Oxford to gain firsthand experience of sperm counting and semen analysis. All three groups have to handle a huge amount of videomicrography data. They share common interests in many aspects of their work, and often engage in collaborative research and knowledge exchange. However, they have different emphases in using videomicrography data. Biomathematicians are particularly interested in identifying different measures that can be extracted from video data, understanding their usefulness in differentiating cells in different categories or conditions, and comparing simulation results with cells captured in experimental videos. Biologists are particularly interested in observing biological phenomena, characterizing them quantitatively, and discovering the relationships between different measurements. Clinical analysts are particularly interested in obtaining the required measurements of a given sample efficiently, performing analysis and making clinical decision accurately, and communicating the results informatively (e.g., to patients).

The deployment of CASA in research and clinical environments is still facing a mixture of challenges ranging from costly equipment to inaccuracy in image analysis. Among these, we identified the following difficulties that could be alleviated by deploying appropriate visualization techniques, which are largely absent from the current provision of CASA.

- Whereas the most common task is to observe and compare different cells in one or a few videos, it is difficult to pay attention to multiple cells concurrently. This phenomenon of "selective looking" has been well studied in cognitive sciences (e.g., [48], [4]). Watching videos repeatedly in order to look at different cells selectively and to build a multi-object mental model for comparison places a high demand on users' time and cognitive load.

- While more and more motion and morphology parameters have been introduced in CASA, domain specialists still rely on aggregated statistics and line graphs for interpreting these parameters. To observe $p$ timevarying parameters of $q$ cells, one needs to visualize $p \times q$ time series plots similar to that shown in Fig. 2(b). Such line graphs can not convey any spatial context, e.g., local density, and collision avoidance.

- Although aggregated statistics remain an effective tool for summarizing observational findings, presenting many different parameters only in numerical values or using basic statistics graphics (e.g., bar charts) may not always be effective in communicating such findings.

These difficulties thereby motivated us to develop new visualization techniques for CASA.

\section{Measurements in Semen Analysis}

In biology research, sperm kinematic and mechanical measures are used to characterize sperm swimming patterns. There are many proposed measurements in the literature. The following sections outline a subset of parameters that were deemed to be the most important indicators of sperm motility. The kinematic measures used in Section 4.1 were defined by Katz and Davis [33] and recommended by the WHO laboratory manual [70]. For an in-depth review of the state-of-the-art in sperm kinematics, we refer readers to [45]. Section 4.2 describes morphology and mechanics measures of a cell head. Section 4.3 gives the mechanical measures of a flagellum. The measures considered here are applicable to human sperm, each with a normal convex head and a single flagellum. For this work, we did not consider rare aberrant morphological cases, such as cells with an 


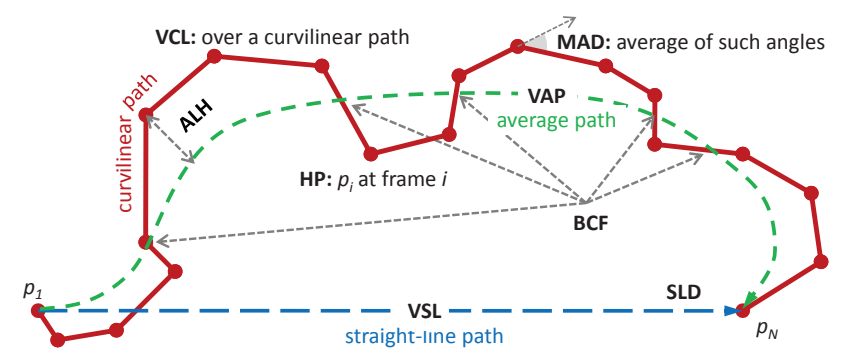

Fig. 3: Some of the observable kinematic measures of a sperm. Among them, VCL, VSL, VAP, ALH, BVF and MAD are defined in the WHO laboratory manual [70].

irregular head shape, multiple heads or multiple flagella. As only a few sperm among millions have fertilization potential, we decided to focus on normal cells in this first application of video visualization to CASA.

\subsection{Kinematic Measures of the Head}

The centroid of a sperm head is typically tracked for a period of 1 second, the standard tracking time defined in [70]. This results in a list of head positions (HP), $p_{i}, i=1,2, \ldots, N$, for the $N$ frames in the period. The list is referred to as a track, or a curvilinear path, which is used to analyze the kinematics of the cell as depicted in Fig. 3. VCL, Curvilinear Velocity $(\mu \mathrm{m} / \mathrm{s})$ : The time-averaged velocity of a sperm along its actual curvilinear path, over $N$ frames. The instantaneous velocity $v c_{i}$ is computed using three consecutive head positions, $p_{i-1}, p_{i}$ and $p_{i+1}$ as:

$$
v c_{i}=\frac{\left\|p_{i}-p_{i-1}\right\|+\left\|p_{i+1}-p_{i}\right\|}{2 \Delta t} .
$$

where $\Delta t$ is the time interval between two consecutive frames. VCL is computed by averaging the instantaneous velocities for the $N$ frames, that is:

$$
V C L=\frac{1}{N} \sum_{i=1}^{N} v c_{i} .
$$

VSL, Straight-Line (Rectilinear) Velocity $(\mu \mathrm{m} / \mathrm{s})$ : The time-averaged velocity of a sperm along the straight line between the end points of its curvilinear path over a standard period.

VAP, Average Path Velocity $(\mu \mathrm{m} / \mathrm{s})$ : The time-averaged velocity of a sperm along its average path. The instantaneous average velocities $v a_{i}$ are computed from the smoothed average path given by the set of points $\bar{p}_{i}=$ $\left(\bar{x}_{i}, \bar{y}_{i}\right)$ and is computed in the same way as $v c_{i}$.

In addition to velocity, angular measures are useful in discriminating between sperm exhibiting widely different swimming patterns [33].

ALH, Amplitude of Lateral Head Displacement $(\mu \mathrm{m})$ : Magnitude of lateral displacement of a sperm head about its average path. It can be expressed as a maximum or an average of such displacements. Different CASA instruments compute ALH using different algorithms, so values may not be comparable among systems.

BCF, Beat-Cross Frequency $(\mathrm{Hz})$ : The average rate at which the curvilinear path crosses the average path.

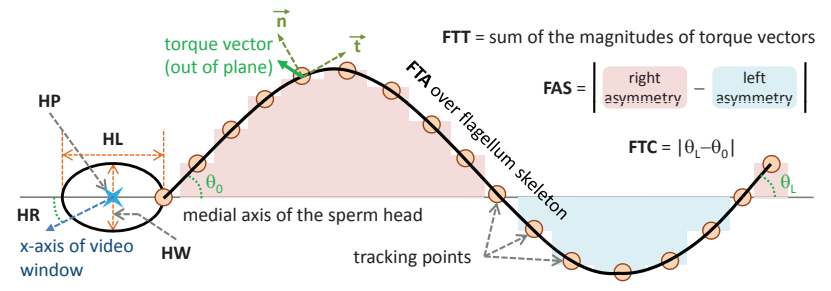

Fig. 4: Sperm measures are computed by fitting geometry to cells extracted from video data. Head morphology (HP, HL, HW, HR) and flagellum mechanics (FTA, FTC, FTT, FAS) are estimated using this proxy geometry.

MAD, Mean Angular Displacement (degrees): The timeaveraged absolute values of the instantaneous turning angle, denoted by $\theta_{i}$, of the sperm head along its curvilinear path.

In addition, a number of abstract measures of the progressiveness of the sperm swimming may also be computed. These include: the straight line direction SLD; the linearity of the curvilinear path LIN, which is the ratio $L I N=V S L / V C L$; the wobble factor WOB, which measures the oscillation of a curvilinear path about the corresponding average path, and is defined as the ratio $W O B=V A P / V C L$; and the linearity of the average path STR, which is given by the ratio $S T R=V S L / V A P$. These measures are used to detect cell motility modes such as hyperactivation.

\subsection{Head Rotation Analysis}

The shape of a sperm head is captured as a set of pixels representing the projected head on the microscope focal plane. As shown in Fig. 4, the length $\mathbf{H L}$ and width $\mathbf{H W}$ of the head, measured in $\mu m$, are estimated by ellipse fitting [21]. This allows us to determine subtle changes in the head's shape and rotation $\mathbf{H R}$ in degrees. These measures are used to detect non-planar beating of the flagellum and encode implicitly the circumference and area of the ellipse. Its centroid is used to track the sperm's location, i.e., HP.

\subsection{Flagellum Mechanics Analysis}

The flagellum of a sperm is a type of eukaryotic flagella. Its regular beating propels the motion of the cell. As shown in Fig. 4, each tail-like flagellum is represented by a polyline and is tracked through the video. The polyline in frame $i$ is stored as a series of points $q_{i, 0}, q_{i, 1}, \ldots$. As the flagellum is almost featureless in the image, it is not possible to determine each point precisely in different frames. When we assume that a sperm is swimming more or less in a 2D space under the microscope, the arclength of the flagellum is a more reliable factor than any feature-based tracking of points on the flagellum. We hence sample each polyline equally to obtain a point set with $L+1$ points. For the following parameters, we first compute them for each frame $i$, and then obtain their average over $N$ frames in a standard period. We thus omit subscript $i$ in the notations of FTA, FTC and FTT for clarity.

FTA, Total Projected Arclength $(\mu \mathrm{m})$ : The total arclength of a flagellum, FTA, is computed as the sum of the 
Euclidean distances $\|*\|$ between the consecutive points:

$$
F T A=\sum_{j=1}^{L}\left\|q_{j}-q_{j-1}\right\|
$$

FTC, Change in Angle (degrees): The difference between the angle of the flagellum at the head $\theta_{0}$ and the angle of the flagellum at the tip $\theta_{L}$. This gives the total amount of sliding undertaken by the flagellum about its centerline as in Fig. 4.

FTT, Total Torque $N \mu$ : The viscous torque represents the tendency of the flagellum to induce rotational motion. Assuming planar beating, the torque is implicitly in the direction out of the beat plane, as shown in Fig. 4. From resistive force theory [25], [42], the viscous drag force per unit length, $F$, exerted by the flagellum on the fluid is:

$$
F=C_{T}(\vec{t} \cdot \vec{u}) \vec{t}+C_{N}(\vec{n} \cdot \vec{u}) \vec{n}
$$

where $C_{T}$ and $C_{N}$ are resistance coefficients. Using the definition by Gray and Hancock [25], $\vec{t}$ is the tangent vector, $\vec{n}$ is normal to $\vec{t}$ in the plane, and $\vec{u}_{j}$ is the rate of change of the flagellum between points $q_{j-1}$ and $q_{j}$. This computation yields a vector whose components describe the viscous drag in the $x$ and $y$ directions. From Equation 3 we can derive the total torque over the flagellum, where $F_{j}^{x}=\left(C_{T}(\vec{t} \cdot \vec{u}) \vec{t}_{x}\right)_{j}$ and $G_{j}^{x}=\left(C_{N}(\vec{n} \cdot \vec{u}) \vec{n}_{x}\right)_{j}$ in the $x$ direction and similarly in the $y$ direction. The viscous torque per unit length is $F T T_{j}=\vec{q}_{j} \times \vec{f}_{j}$ and $\vec{f}_{j}=\left[F_{j}^{x}+G_{j}^{x}, F_{j}^{y}+G_{j}^{y}\right]$. The total viscous torque that the flagellum exerts on the fluid is the magnitude $|*|$ of the sum of vector components:

$$
F T T=\operatorname{abs}\left(\left|\sum_{j=1}^{L}\left(\vec{q}_{j}^{x} \vec{f}_{j}^{y}-\vec{q}_{j}^{y} \vec{f}_{j}^{x}\right) \delta s\right|\right)
$$

We use only the absolute value of FTT as a measure since its sign correlates with the asymmetry.

FAS, Asymmetry: The asymmetry of the flagellum about the medial axis of the head is a standard indicator of hyperactivated swimming, which is functionally critical for successful fertilization and is required to penetrate the oocyte. As illustrated in Fig. 4, asymmetry is measured by integrating the amplitudes of the flagellum about the medial axis of the head. The equations outlined above are applicable to any eukaryotic flagella. The physical behaviors of other types of flagella in bacteria, such as prokaryotic flagella, are different in general.

\subsection{Uncertainty in Sperm Cell Extraction}

The video data considered in this work was captured using phase contrast microscopy. The video processing algorithms had to deal with a number of technical challenges in object recognition, segmentation and tracking because of background noise, debris, and close proximity of flagella in each frame. It is thus necessary to convey the potential errors in video processing to the users. We thus introduced uncertainty measures at different stages of the video processing pipeline. Image processing techniques, such as segmentation and morphological operations facilitate
TABLE 1: A total of 20 measures discussed in Section 4 and their corresponding computational characteristics.

\begin{tabular}{lcccc} 
measure & type & semantics & frame & interval \\
\hline HP & $\mathbb{R}^{2}$ & position & yes & - \\
SLD & $\mathbb{R}^{2}$ & direction & yes & average \\
VCL & $\mathbb{R}$ & velocity & - & yes \\
VSL & $\mathbb{R}$ & velocity & - & yes \\
VAP & $\mathbb{R}$ & velocity & - & yes \\
ALH & $\mathbb{R}$ & distance & yes & average \\
BCF & $\mathbb{R}$ & counting & - & yes \\
MAD & $\mathbb{R}$ & angle & yes & average \\
LIN & $\mathbb{R}$ & ratio & - & yes \\
WOB & $\mathbb{R}$ & ratio & - & yes \\
STR & $\mathbb{R}$ & ratio & - & yes \\
HL & $\mathbb{R}$ & length & yes & average \\
HW & $\mathbb{R}$ & width & yes & average \\
HR & $\mathbb{R}$ & angle & yes & average \\
FTA & $\mathbb{R}$ & length & yes & average \\
FTC & $\mathbb{R}$ & angle & yes & average \\
FTT & $\mathbb{R}$ & torque & yes & average \\
FAS & $\mathbb{R}$ & balance & yes & average \\
UH & range $[0.0,1.0]$ & uncertainty & yes & average \\
UF & range $[0.0,1.0]$ & uncertainty & yes & average \\
\hline
\end{tabular}

target tracking of sperm heads. We estimate an uncertainty measure (UH) for all processing related to a sperm head, and another measure (UF) for all processing related to a sperm flagellum. The measurements are computed based on the following factors:

- the distinction of an object (head or flagellum) from detractors (noise, debris) may be uncertain;

- the tracking algorithm may fail to recognize an expected and previously recognized object;

- the tracking algorithm may be confused by multiple, similarly-moving objects;

- the segmentation of an object may yield a significantly different set of measures (e.g., flagellum length, pixel occupancy) in comparison with an expected object or previously tracked and segmented object.

These two uncertainty measures, UH and UF, are visualized in conjunction with the other measures discussed in the previous subsections.

\section{Off-the-Shelf Design Options}

In Section 4, we described a total of 20 measures, some of which can be obtained at individual frames, and some can be computed over a temporal interval. They are all represented by real numbers but vary in their basic semantic meaning. Table 1 summarizes some computational characteristics of these measures. From the task analysis described in Section 3.2, we can conclude that biomathematicians would like to discover what measures are important, and biologists would like to have the flexibility to observe different measures for different videos or different hypotheses. Meanwhile, clinical analysts would prefer to be informed of a standard set of parameters and a standard visual representation. We concluded that the requirement of clinical analysts can only be met after a long period scientific and technological development. It was more realistic to address the needs of biomathematicians and biologists first. 
The biomathematicians and biologists have been using time series plots to depict those measures computed over a regular interval. Fig. 2(b) shows an example of such a plot. Hence in brute force, one can visualize the motion characteristics of each sperm using one scatter plot for HP and SLD and some 18 time series plots for the others. To compare $q$ cells, one can juxtapose $q$ sets of measures in each of the 19 plots. Whereas such visual representations are useful for observing different measures individually, they do not provide adequate support for multivariate observation and reasoning. Not only is there a huge cognitive load in visual search for individual measures and in assembling a mental picture for the motion characteristics of each sperm, but also an absence of spatial context in viewing the time series plots.

One alternative visual representation is parallel coordinate visualization, which may have 20 real axes for the 19 interval-based measures with a total of $\mathbb{R}^{20}$ dimensionality. The measure of each sperm can be displayed as a line across different axes. Fig. 2(c) shows an example of such a plot for a specific time interval. For multiple time intervals, $T_{1}, T_{2}, \ldots$, one can make use of additional parallel coordinate visualizations, each encoding measures in a specific time interval $T_{i}$. It is not difficult to observe the shortcoming of such visual representations. There is a complete absence of spatial context, while it is a cognitive burden in searching for different measures. Users will also be required to try different ordering of axes to make observations about the correlation between various measures. Furthermore, when using multiple parallel coordinates to depict measures in a series of time intervals, it would be very difficult to observe temporal behavioral changes of individual cells.

\section{Glyph-based Visual Design}

Following the consideration of two off-the-shelf design options in Section 5, we adopted glyph-based visualization. We divide video frames into a series of temporally-ordered subsets of a pre-defined reference period, $T_{\text {ref }}=1$ second, as recommended in [70]. Hence, the motion characteristics of each sperm can be computed in regular intervals defined by $T_{r e f}$, and the statistical measurements in each interval can be mapped to a glyph. For a sperm captured in a video, the series of temporal subsets of frames enables the sperm to be depicted as a series of glyphs as in Fig. 2(d). Several domain specialists (who are also co-authors) were actively involved in the glyph design process. They made several design recommendations.

1) It is desirable to encode some 20 measures in a single glyph. This presents a huge challenge as all measures are numerical rather than categorical, while the existing works in the literature have so far attempted up to 12 attribute dimensions.

2) It is undesirable to assign visual channels based on an importance ordering of these measures, since one of the visualization tasks is for biomathmaticians to discover the importance of individual measures in analyzing different motion features. Biologists made the same recommendation as imposing an importance ordering may introduce unnecessary bias in favor of a subset of phenomena and known hypotheses, while handicapping observation of other phenomena and generation of new hypotheses. The absence of an importance order results in the lack of definite guidelines about selecting visual channels according to the order of their discriminative capacities [44].

3) Some measurements are related (e.g., VSL, VCL, VAP, ALH, etc.) and there is a need to support comparative analysis for these measures within the context of a single sperm.

4) It is desirable for the visual design to convey the visual signatures of some major classes of cells, such as live vs. dead, human sperm vs. mouse sperm.

5) It is desirable for the visual design to facilitate effective perception and cognition. Although it is not an essential requirement to be able to judge numerical values accurately (as glyphs will not be the best tool for such tasks), it would be helpful that each attribute identified can be individually compared across many glyphs spatially and temporally.

6) Some domain specialists expressed a wish to have a glyph resembling a sperm, which poses an interesting challenge. While it indicates the importance for the visual representation to exhibit some metaphoric design features, it is a non-trivial requirement as the realistic representation of a sperm, especially its bendy flagellum, was a causal factor for the difficulties to observe motion characteristics.

We made use of the glyph design principles and the relevant literature in [44]. Here we only discuss the most relevant ones to avoid repetition. In perception, there is a rich collection of studies on integral and separable dimensions of visual channels. In general, given two visual channels with dissimilarity of $d_{a}$ and $d_{b}$, the combined dissimilarity of an integrated design is about their Euclidean distance $\sqrt{d_{a}^{2}+d_{b}^{2}}$ [37], [26]. On the other hand, the combined dissimilarity of separable visual channels is close to their city-block distance $d_{a}+d_{b}$ [9], [60]. We adopted the latter concept as the most important design principle in this work. To enable different attribute dimensions to be mapped onto separable visual channels, we first tried to encode different dimensions using spatially independent lines or shapes. Secondly, in some cases where we had to use the same line or shape to encode more than one attribute in order to reduce clutter, we ensured that the host shape would never disappear because one attribute value was too small. Thirdly, we did not use different color dimensions (e.g., RGB or HSV) to encode different attributes. We avoided using opacity in our encoding since it could affect color perception.

We started our design activities with a series of brainstorm meetings. We produced swatch charts for the major designs, and evaluated their suitability by considering issues such as visual channel separation, channel conflicts, scalability, compactness, clarity, attention bias, comparability, 


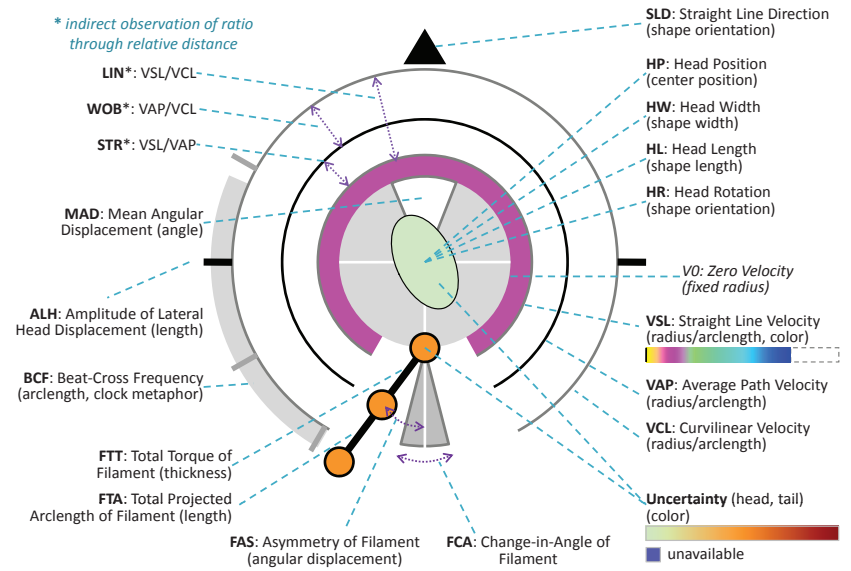

Fig. 5: Glyph Schematic: this schematic shows how 20 parameters are explicitly encoded into the the glyph. Parameters such as LIN, WOB and STR are implicitly represented by how the velocity parameters are laid out in the glyph.

and metaphoric abstraction. We used multi-scale 'crash test' to ensure that glyphs would still be legible at the lowest resolution. An example swatch chart and a multi-scale crash test can be seen in the Appendix. We presented several initial designs to the domain specialists and sought their opinions. We found that the domain specialists responded better to more abstract glyph designs that made use of simple lines and shapes.

After numerous iterations, occasionally involving debates and ranking exercises, we eventually converged into a design as illustrated in Fig. 5. The design encodes a total of 20 attribute dimensions directly. These include 6 WHO-recommended measures for head kinematics (VCL, VAP, VSL, BCF, ALH, MAD), 5 further head measures (HP, SLD, HW, HL, HR), 4 flagellum measures (FTA, TCA, FAS, FTT), and 2 uncertainty measures in processing the head and flagellum data. In addition, 3 other WHOrecommended measures (LIN, WOB, STR) can be observed indirectly by comparing VCL, VAP and VSL.

As this is a new approach in semen analysis, there were a number of challenges and sometimes conflicting recommendations, some which are discussed below.

The three velocity measurements, i.e., curvilinear (VCL), average path (VAP) and straight line (VSL), are the most used attributes in semen analysis. The visual comparison of these measurements is also necessary for observing three ratio factors, $L I N=\frac{V S L}{V C L}, W O B=\frac{V A P}{V C L}$ and $S T R=\frac{V S L}{V A P}$. We thus map these three attributes to the radii of three open-ended arcs. This avoids the arcs being interpreted and compared as areas. As radii are linearly related to the circumferences, the line lengths offer equivalent but more scalable visual quantities. Metaphorically, velocity is associated with distance or length; hence these three mappings are easy to remember. Although we always have the spatial relationships $V C L \geq V A P \geq V S L$, we further improve their identification by assigning a much darker color to the middle line, VAP. The geometrical mapping of these three velocities is defined as:

$r_{v c l}=\frac{V C L+C_{\text {base }}}{C_{\text {scale }}}, r_{\text {vap }}=\frac{V A P+C_{\text {base }}}{C_{\text {scale }}}, r_{\text {vsl }}=\frac{V S L+C_{\text {base }}}{C_{\text {scale }}}$

where $C_{\text {base }}$ and $C_{\text {scale }}$ are two user-definable constants. $C_{\text {scale }}$ specifies the overall scaling factor of the glyph in relation to microns $\mu \mathrm{m}$, the standard distance unit in semen analysis. For example, if $100 \mu \mathrm{m}$ is mapped to 50 pixels, $C_{\text {scale }}$ is set to 2 . $C_{\text {base }}$ specifies the minimal glyph radius to prevent the generation of glyphs that are too small to see. Since the velocity of a sperm affects the overall glyph size, a sperm that is not in motion could potentially be mapped to a tiny dot in the visualization. One normally sets $C_{\text {base }}$ in relation to the maximum head length of the sperm to be studied. For glyphs in this paper, we set $C_{\text {base }}=80 \mu \mathrm{m}$ and $C_{\text {scale }}=5$.

Normally, the ratio between VSL and VCL (i.e., LIN) varies between 0.2 and 0.8 . However, sometimes it may become very small, at the scale of 0.01 . Visually it will be difficult to distinguish between the VSL of such a sperm and the VSL of a dead one (note: there is little risk in mistaking a live one for a dead one as VCL and VAP would be large enough). To make VSL more perceptible, we add a redundant color channel for the circular strip between VSL and V0. This is one of three colored regions in the glyph; hence it is fairly noticeable. The colormap for VSL is shown in Fig. 6, which consists of six bands. While overcoming the visibility problem of VSL, this also addresses requirement (4).

Since the arc for VSL is not an associative visual channel [5], a colormap was also designed to facilitate metaphoric color symbolism (e.g., pink pig, grass-eating ram and oceanic sperm whale) to aid memorization (Fig. 6). The links among colors, languages, memorization and communication accuracy were studied extensively in psychology and anthropology. For example, Lucy and Shweder [43] confirmed that the combination of color and linguistic description aids both short- and long-term memory. Stokes [63] confirmed the positive effort of using metaphoric colors to aid learning. Hence encoding VSL using both arc length and metaphoric colors. The former facilitates quantitative visual search and reasoning at a reasonable resolution, and the latter learning and memorization. The colormap is divided into three sections. The first section between 0 and $100 \mu \mathrm{m} / \mathrm{s}$ covers the majority needs in observing and analyzing human semen samples. The combination between the first and second sections, with a range from 0 to $300 \mu \mathrm{m} / \mathrm{s}$, comfortably covers all VSL measures that we have found in the literature. We assigned white to any VSL above $300 \mu \mathrm{m} / \mathrm{s}$ for any unexpected new discoveries.

Kinematic measure BCF (beat-cross frequency) created some difficulties for us as it encapsulates the number of interactions between VCL and VAP. We did consider several design options of using dots or short lines to represent $\mathrm{BCF}$. However, because $\mathrm{BCF}$ varies between 2 and $35 \mathrm{~Hz}$, it will be highly inefficient to rely on object counting as a visual mapping [68]. We thus mapped BCF to a shaded circular strip between VCL and VAP as shown in Fig. 5. 


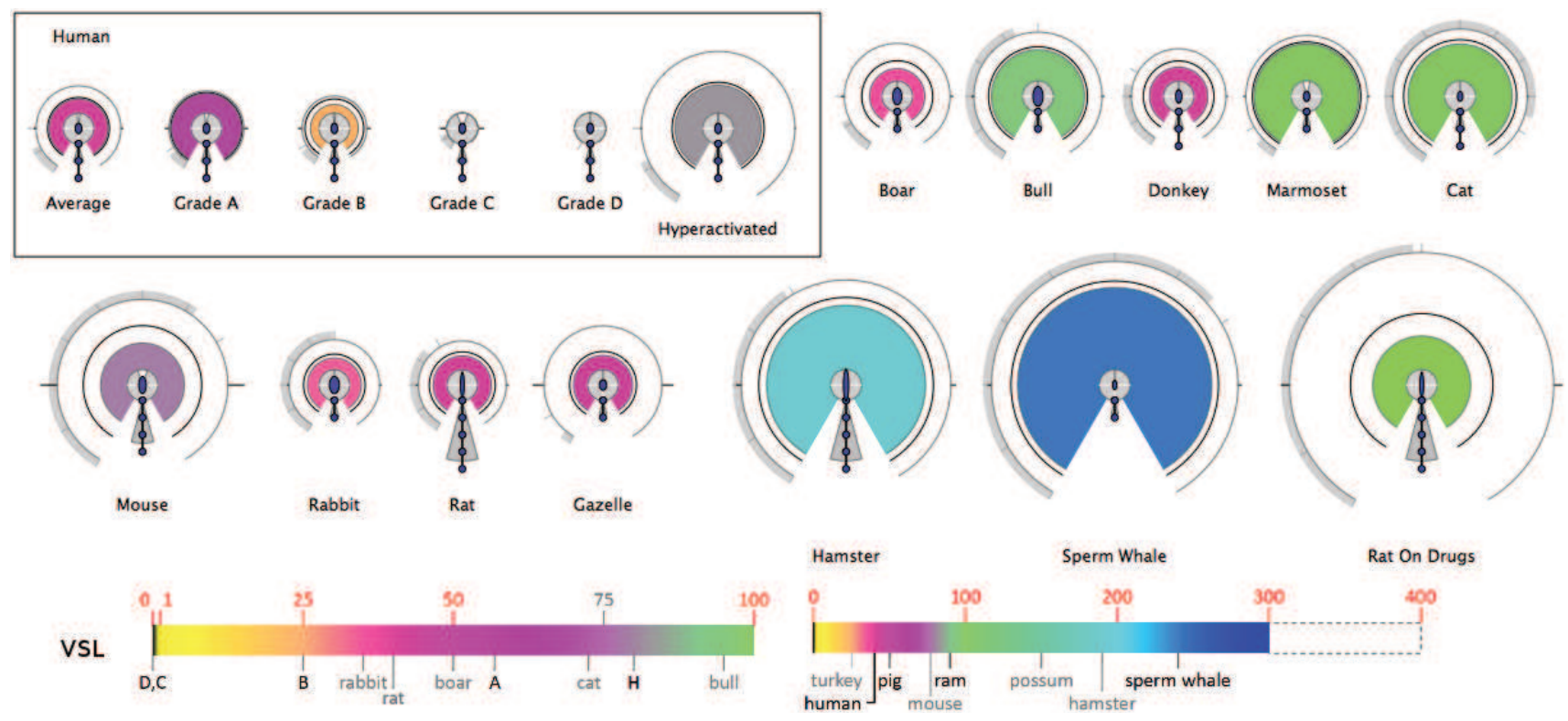

Fig. 6: The average sperm glyphs for multiple species. Although VSL is a continuous measure, the average grades for human sperm cells and some mammals are marked on the VSL colormap as reference points. This facilitates learning and memorizing of the colormap, and serves as a reference for inter-species comparison. Measures for all species shown were gathered from the research literature. The highest VSL values found are those of sperm whales. Unexpected high values are encoded as white.

It makes use of a common partition of a circumference into 6 sections of 10. With open arcs of VCL and VAP, 5 sections are used, covering a range $0-50 H z$. BCF can easily be estimated as one would estimate minutes on an analogue clock. Starting from the 7 o'clock position $(\mathrm{OHz})$, it is not difficult to identify values $5 \mathrm{~Hz}$ (8 o' clock), $10 \mathrm{~Hz}$ (9 o'clock), and so on. ALH and SLD offer additional markers for 10,25 and $40 \mathrm{~Hz}$.

A new measurement, FTT (total torque of flagellum) was another attribute that brought about many contentious design options. After many iterations, we decided to map it to the thickness of the pendulum line that was originally designed for representing FAS (flagellum asymmetry). Our initial instinct was to avoid loading two attributes onto the same object. However, after careful analysis we realized that the two visual channels can still be considered separable as the perception of thickness does not depend on the angle of FAS (assuming effective anti-aliasing in rendering) and vice versa. It is necessary to ensure that the pendulum line will never disappear [22]. We achieve this by (a) setting its minimal thickness to a single pixel, (b) setting its length the same as the radius for VCL, (c) always displaying the black pendulum head even if VCL $=0$, and (d) assigning the highest display priority so it will not be occluded by other components of the glyph.

In addition, we made a number of metaphoric associations to help domain specialists remember the coding scheme, such as "beak" as a directional pointer (SLD), "big mouth" for "madness" (MAD or mean angular displacement), "pendulum" for imbalance (i.e., asymmetry or FAS), and "tail" for two of the major attributes of flagellum.

\section{Application Scenarios}

Scientists in semen analysis routinely deal with video data when observing sperm samples. They obtain multivariate measures in analyzing cells, e.g., assessing cell fitness and function. In the long term, glyph-based video visualization is applicable to toxicology, pharmacology, fertility, animal husbandry, and the design of micro devices for sperm selection and sorting in IVF. This work represents the first step in a research environment. Usually, video data captured for research is of a better quality than that captured in a clinical environment. The videos used in this work represent a typical application scenario, where researchers need to observe and analyze sperm in different experimental conditions. For example the two videos used in this paper feature sperm suspended in fluid with different viscosities. Using a more viscous fluid slows the sperm progression, allowing researchers to observe and analyze flagellar beating patterns more easily. The experiments were related to the longterm research questions of the domain specialists involved in this work. These questions include: what characterizes normal motility, and how do the good sperm that make it through the cervix swim? Answering this question would provide the baseline for assessing the effect of drugs, toxic effects, temperature, $\mathrm{pH}$, etc., but would also require many experiments, video capturing, viewing and processing, and computing and observing a variety of measures. Although answering such general questions is beyond the scope of this paper, domain specialists selected three sperm from the two videos and suggested several specific questions as prompts for glyph-based video visualization:

- Can we see what the cells and their flagella do kinematically and mechanically as these are difficult 
to note down from watching videos?

- Which kinematic measure may indicate dysfunction?

- Which mechanical measures indicate dysfunction?

- What ranges of values characterize normal function?

- What behaviors are more observable in a high (or low) viscosity medium?

Our approach affords researchers an effective means to visualize 20 explicit and 3 implicit parameters for each sperm simultaneously in a static scene, and alleviates the demanding need for frequently switching between viewing videos and simple statistics plots in order to observing different measures, different cells, different spatial and temporal contexts. Scientists found two main uses of our glyph-based visualization, namely, summary glyphs (Section 7.1) and sperm tracks (Section 7.2). While these two videos are not sufficient to make any substantial claims in biology, they clearly demonstrate that the glyph-based video visualization allows scientists to observe their experimental data more easily, reducing the demand on time, and likely cognitive load, in analyzing multivariate data.

\subsection{Summary Glyphs}

One application of semen analysis is in inter-species comparison of cell function. The comparison makes uses of average values of a set of parameters for sperm cells as discussed by Holt et al. [29]. Ultimately they are used for toxicology, andrology and designing micro devices for cell selection, as well as for scientific education. Motility data for different mammals was collected from a number of sources in the biology literature [30], [54], [39], [51], [64], [18], [1]. Cummins et al. [13] provided a comprehensive list of average mammalian sperm motility measurements.

Fig. 6 shows a set of summary glyphs encoding a number of kinematic measures for cells of different species. For the flagellum only arclength is encoded by the glyphs as the other parameters were not available in the literature. To indicate this, the uncertainty of the flagellum is set to unavailable. Fig. 6 provides an effective visualization of the differences in sperm behavior across species. The grading system for human sperm cells from the WHO manual [70] is as follows: Grade A: rapidly progressive motility; Grade B: slow or sluggish progressive motility; Grade $C$ : non-progressive motility; Grade D: immotility; Grade $H$ : hyperactivated sperm, exhibiting asymmetric and irregular beat patterns, and high velocities associated with fertilization and penetration of the oocyte. In conjunction with the colormap it is easy to compare velocities of sperm. For example, the "Rat On Drugs" dosed with EGEE (ethylene glycol monoethyl ether), a toxin that is known to affect sperm motility, has high VCL and medium VSL indicating less progressive erratic swimming; while the sperm whale shows that fastest VSL with slower VCL than the dosed rat indicating more progressive swimming. The sperm whale in comparison shows a lower VSL to VCL ratio than the dosed rat, despite having lower velocity; this indicates more progressive swimming.

In addition, the domain specialists provided us with a collection of videos, two of which are featured in this paper.

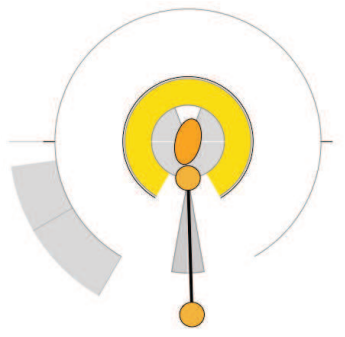

(a)

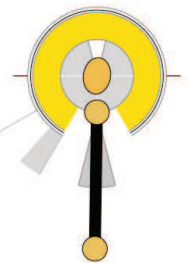

(b)

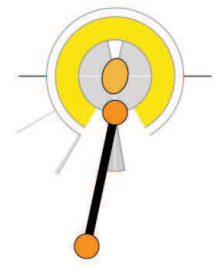

(c)
Fig. 7: Summary glyphs comparing (a) sperm in a low viscosity medium with $(b, c)$ those in a high viscosity medium.

Video A, which features a single sperm cell from a normal research donor, was captured with an Olympus BX-50 microscope, positive phase contrast and Hamamatsu C9300 CCD Camera at a framerate $293.4 \mathrm{~Hz}$, pixel size $7.4 \mu \mathrm{m}$ at 20x magnification. The medium is Earle's balanced salt solution with sodium lactate, sodium pyruvate, without phenol red and viscosity increased with Sigma M7140 Methylcellulose $2 \%$, nominal viscosity 15 cPoise with no stage heater (ambient temperature). The sperm had migrated into a $400 \mu \mathrm{m}$ depth, $4 \mathrm{~mm}$ width capillary Kremer test assay. The sperm in the image was at approximately $2 \mathrm{~cm}$ migration distance.

Video B, which features many sperm cells, was filmed under similar conditions except at a lower framerate $50 \mathrm{~Hz}$. Human tubal fluid, an IVF medium, was used with viscosity increased with Sigma M0262 Methylcellulose 2\% (nominal viscosity $400 \mathrm{cPoise}$ ). The sperm had migrated $1 \mathrm{~cm}$ into a $400 \mu \mathrm{m}$ depth, $4 \mathrm{~mm}$ width capillary Kremer test assay.

Fig. 7 shows the summary glyphs for three cells selected from these two videos. Fig. 7(a) shows the summary glyph of the sperm cell in Video A (low viscosity), which appears to be a straight swimmer based on the angular displacement of the tail pendulum (FAS). However, it shows a high VCL in comparison to VAP and VSL, indicating an erratic swimming pattern. This could be misinterpreted as non-progressive swimming without visualizing VCL, VAP, VSL, and FAS together. The two glyphs in (b) and (c) are for two cells in Video B (high viscosity), showing much more progressive swimming patterns by exclusively examining the velocities. The tail pendulum of Fig. 7(b) indicates a possible straight swimmer, but that of Fig. 7(c) shows a consistent angular displacement, suggesting an unusual swimming pattern.

\subsection{Glyph Tracks}

Whilst summary glyphs are useful for observing overall statistical differences, glyph tracks enable scientists to observe individual sperm with spatial and temporal details. Fig. 8 shows the glyphs tracks of the same three cells in Fig. 7. Here one glyph is rendered for each second of the video. The blue track path in Fig. 8(a) shows the erratic swimming pattern of the sperm in the low viscosity medium (Video A). This erratic behavior is consistent over time as all three glyphs along the track show a large radial distance between 


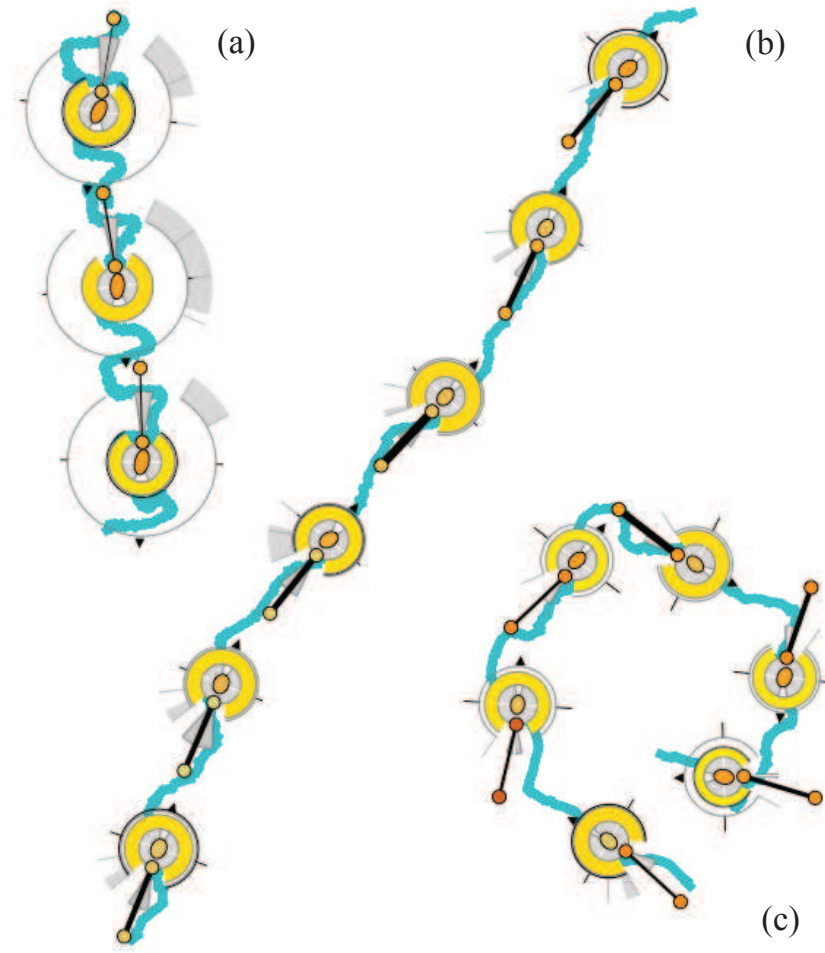

Fig. 8: Glyph tracks enable more detailed observation and analysis of the three cells in Fig. 7.

VSL and VCL in comparison with glyphs on the two other tracks in (b) and (c). When comparing the tracks in (b) and (c) for the two sperm in Video B, the reason of the angular displacement shown in Fig. 7(c) is immediately obvious. The sperm in (c) is actually swimming in circles and is considered dysfunctional. When examining the glyphs along the track closely, the sperm in (b) exhibits small periodic changes in parameter values across the track, while that in (c) shows less consistency along the track, such as aperiodic fluctuation in VCL and VAP. One can observe that the circular swimmer in (c) shows higher ALH and lower BCF than the straight swimmer in (b). The flagellum torque (FTT, pendulum thickness) of the circular swimmer also fluctuates aperiodically, though this may be due to less reliable image processing for the flagellum as the uncertainty in flagellum tracking appears high.

The glyph tracks can be utilized as a means of external memorization of the video. Fig. 2(d) summarizes the video data of the high viscosity medium (Video B). Note that the image processing stage of the software has filtered out most debris and unreliable tracks. For those image processing errors that has not been algorithmically detected (e.g., the large purple glyph), human observers can easily filter them out based on their tacit knowledge.

\subsection{Implementation}

We used Matlab for implementing the image processing stage of the system, and Python, PyQt4 and SciPy for visual mapping and user interface. The visualization stage can handle the multivariate and multi-cell data, such as Video B at an interactive frame rate. The computational bottleneck is the semi-automated image processing stage. All glyphs are rendered with vector graphics to maintain the display quality. The code for visual mapping and glyph rendering is given in the supplementary materials.

\section{Evaluation by DOMAIN EXPERTS}

As part of an iterative process [47] for task analysis, visual design, software development and evaluation as mentioned in Section 3.2, much of the evaluation was carried out in informal settings, such as brainstorm meetings, placement, emails and phone calls. In this section, we give a snapshot of evaluation conducted in relatively formal settings. In addition to close interaction with the three domain experts in the team, we interviewed domain experts in formally organized evaluation meetings. These meetings focused on a number of avenues for potential uses of glyph-based video visualization to (a) alleviate the need for watching videos repeatedly, (b) summarize multiple measures of sperm under investigation, (c) help scientists comparing different cells in the same video or different videos, and (d) help clinical analysts in routine semen analysis.

Different forms of evaluation collectively confirmed the above-mentioned potentials in (a), (b) and (c). However, there was no evidence to support the potential of (d). We briefly described the discussions in the formally organized meetings after the visualization system was implemented.

Meetings with Clinical Analysts. In a half-day meeting, we sought advice and feedback from two clinicians at the Institute of Reproductive Sciences in Oxford. After our presentation, the discussion quickly shifted to the existing systems for semen analysis. We were informed that the current clinical practice focuses mainly on sperm counting. While computerized systems can automatically return meaningful numbers of sperm concentration, clinicians are fully aware of the fact that they are not accurate, especially when samples contain blood cells, bacteria, debris and other artifacts. Manual counting is a routine operation for embryologists in the Institute. An embryologist processes approximately 20 samples per day. There is an overwhelming demand for more accurate and cost-effective counting system. We realized that the domain experts mistook the visualization system as a computer vision system. We explained that the visualization is designed to supplement and complement the automatic vision system. Once the discussion was re-focused back to the use of visualization in semen analysis, the clinicians immediately suggested that it would be very useful to researchers. We concluded that the visualization is not ready to support clinical semen analysis as the transition from research techniques to clinical practice would normally take some time.

On the other hand, the two clinicians noted that glyphbased visualization would be useful for validating measurements obtained using automatic systems, and could also be used as a record for manual evaluation processes. They particularly liked our approach for considering both head and flagellum, as the inaccuracy in many existing systems 
may result from counting the head only. The fact that the visualization can allow scientists and researchers to observe many measures at the same time will be a big bonus. This indicated a potential for using visualization to help domain experts to make use of more measurements.

We explicitly sought advice from the clinicians as a means for improving the visualization. They pointed out that when two trajectories intersect with each other, it was difficult to ascertain whether there was a collision. The team took the problem of trajectory intersection back, and examined several design options, including the two suggestions by clinicians: 3D and opacity-based depth cue. Finally, we converged to a solution that uses a temporal colormap for displaying trajectories as shown in Fig. 2(d).

Meetings with Cell Biologists. We organized a half-day consultation meeting with five domain experts (including cell biologists and biomathematicians) in Experimental Medicine at the University of Birmingham. This was followed by continuing interaction over six months for various software refinements. All domain experts appreciated that the visualization method developed in this work can alleviate the need for watching videos repeatedly. They also appreciated most aspects of our visual design, and agreed that it conveyed more information than any conventional techniques. The experts were enthusiastic about the glyphs and regarded them as useful and metaphorically meaningful. In terms of using three circular arcs to represent VSL, VCL and VAP, the domain experts found that they are rather effective in comparison with any alternatives such as straight lines or color mappings. They considered the possibility of confusing with area mapping not really an issue as professionals could learn to interpret new visual representations quickly. There were discussions among the domain experts over which parameters should be encoded and with what priority. The meeting concluded that it was better to maintain the generality of the current design.

Among all of the visualizations we presented, the domain experts found the aggregated glyphs, such as those in Fig. 6, the most informative. Experts agreed that video summarization such as Fig. 2(d) encapsulated a substantial amount of information in a video, and could be used as a reminder as to what was in the video (i.e., external memorization). However, they found it cognitively more demanding to interpret than glyphs on a single track (e.g., Fig. 8). As there were many glyphs in a summary visualization, some experts wondered if it was possible for important changes to be emphasized visually. However, the meeting could not agree on how to define important changes as they would be dependent on many factors. The meeting also concluded that although glyph-based visualization is a novel and useful technique, it cannot totally replace conventional techniques such as time series plots. Meanwhile, all experts agreed that visualizing several time series plots for each measure or each sperm would not scale up to many sperm captured on a video. The domain experts made a few suggestions of minor refinement of the visual designs, which were incorporated into the results presented in this paper. The experts also outlined some challenges in automatic detection and summarization of behavior changes of sperm, and clustering of similar sperm. These are beyond the scope of this paper.

\section{Conclusions}

We have developed a novel glyph-based video summarization technique for supporting semen analysis in a research setting. We have made use of segmented sperm heads and flagella to compute a large set of kinematic, morphological and mechanical characteristics. We have addressed the demanding needs for encoding 20 dimensions by making use a compact glyph design, which enables users to observe multivariate measures of different cells in a combinatory manner or comparative manner. We have demonstrated that glyph-based video visualization can reduce the need for scientists to watch videos repeatedly, while revealing multivariate patterns and changes in sperm motility that were not easily observable previously from videos or individual time series plots.

We have gained valuable experience in this collaborative research. In particular, we have found that an iterative process of task analysis, visual design, and evaluation is highly effective, allowing mutual knowledge transfer between visualization researchers and domain experts. While we have successfully stretched the capability of glyph encoding in terms of the number of attribute dimensions and deployment in video visualization, we have not identified a concrete set of generic guidelines that can be applied to other glyph design problems. We did find that the different design considerations, criteria and guidelines discussed in [66], [7], [44] are useful. For future work, we will examine means for improving measurement accuracy in processing clinical videos by making use of advanced visual analytics techniques. We also plan to explore such new application avenues. as the methodology developed in this work is applicable to other types of flagella-propelled cells.

\section{ACKNOWLEDGMENTS}

The authors wish to thank Akanksha Mishra and Celine Jones from the Institute of Life Sciences Oxford for the invaluable advice and feedback during the design process. We are also grateful to Drs. David Foo and Hermes Gadêlha for discussions at Centre for Human Reproductive Science, Birmingham Women's NHS Foundation Trust. Imaging data were captured as part of DJS's Fellowship (Medical Research Council grant G0600178). This visualization work was supported by the King Abdullah University of Science and Technology, UK EPSRC, and the James Martin Foundation.

\section{REFERENCES}

[1] T. Abaigar, W. V. Holt, R. A. P. Harrison, and G. del Barrio. Sperm subpopulations in boar (sus scrofa) and gazelle (gazella dama mhorr) semen as revealed by pattern analysis of computer-assisted motility assessments. Biology of Reproduction, 60(1):32-41, 1999.

[2] R. P. Amann and D. F. Katz. Reflections on CASA after 25 years. Journal Of Andrology, 25(3):317-325, 2004. 
[3] S. A. Baba and Y. Mogami. An approach to digital image analysis of bending shapes of eukaryotic flagella and cilia. Cell Motility, 5(6):475-489, 1985

[4] R. Becklen and D. Cervone. Selective looking and the noticing of unexpected events. Memory and Cognition, 11:601-608, 1983.

[5] J. Bertin. Semiology of graphics: diagrams, networks, maps. University of Wisconsin Press, Madison, WI, 1983.

[6] R. Borgo, M. Chen, B. Daubney, E. Grundy, H. Jänicke, G. Heidemann, B. Höferlin, M. Höferlin, D. Weiskopf, and X. Xie. State of the art report on video-based graphics and video visualization. Computer Graphics Forum, 31(8):2450-2477, 2012.

[7] R. Borgo, J. Kehrer, D. H. S. Chung, E. Maguire, R. S. Laramee, H. Hauser, M. Ward, and M. Chen. Glyph-based visualization: Foundations, design guidelines, techniques and applications. In Eurographics, State of the Art Reports, 2013.

[8] R. P. Botchen, S. Bachthaler, F. Schick, M. Chen, G. Mori, D. Weiskopf, and T. Ertl. Action-based multifield video visualization. IEEE Transactions on Visualization and Computer Graphics, 14(4):885-899, 2008

[9] B. Burns, B. E. Shepp, D. McDonough, and W. Wiener-Ehrlich. The relation between stimulus analyzability and perceived dimensional structure. Psychology of Learning and Motivation, 12:77-115, 1978.

[10] M. Chen, R. Botchen, R. Hashim, D. Weiskopf, T. Ertl, and I. Thornton. Visual signatures in video visualization. IEEE Transactions on Visualization and Computer Graphics, 12(5):1093-1100, 2006.

[11] C. D. Correa and K.-L. Ma. Dynamic video narratives. ACM Transactions on Graphics, 29(3):88, 2010.

[12] R. A. Crawfis and M. J. Allison. A scientific visualization synthesizer. In Proc. IEEE Visualization, pages 262-267, 1991.

[13] J. M. Cummins and P. F. Woodall. On mammalian sperm dimensions. Journal of Reproductive Fertility, 75(1):153-175, 1985

[14] G. Daniel and M. Chen. Video visualization. In Proc. IEEE Visualization, pages 409-416, 2003

[15] W. C. de Leeuw and J. J. van Wijk. A probe for local flow field visualization. In Proc. IEEE Visualization, pages 39-45, 1993.

[16] P. Dollár, V. Rabaud, G. Cottrell, and S. Belongie. Behavior recognition via sparse spatio-temporal features. In VS-PETS, pages 65-72, 2005.

[17] P. Dragicevic, G. Ramos, J. Bibliowitcz, D. Nowrouzezahrai, R. Balakrishnan, and K. Singh. Video browsing by direct manipulation. In Proc. ACM CHI, pages 237-246, 2008.

[18] E. Flores, E. Taberner, M. M. Rivera, A. Peña, T. Rigau, J. Miró, and J. Rodríguez-Gil. Effects of freezing/thawing on motile sperm subpopulations of boar and donkey ejaculates. Theriogenology, 70(6):936-945, 2008

[19] R. Fuchs and H. Hauser. Visualization of multi-variate scientific data. Computer Graphics Forum, 28(6):1670-1690, 2009.

[20] H. Gadelha, E. A. Gaffney, D. J. Smith, and J. C. Kirkman-Brown. Nonlinear instability in flagellar dynamics: a novel modulation mechanism in sperm migration? Journal of Royal Society Interface, 7:1689-1697, 2010.

[21] W. Gander, G. H. Golub, and R. Strebel. Least-squares fitting of circles and ellipses. BIT, (34):558-578, 1994.

[22] W. R. Garner. Interaction of stimulus dimensions in concept and choice processes. Cognitive Psychology, (8):98-123, 1976.

[23] A. Girgensohn, D. Kimber, J. Vaughan, T. Yang, F. Shipman, T. Turner, E. Rieffel, L. Wilcox, F. Chen, and T. Dunnigan. Dots: support for effective video surveillance. In Proc. International Conference on Multimedia, pages 423-432, 2007.

[24] D. B. Goldman, B. Curless, S. M. Seitz, and D. Salesin. Schematic storyboarding for video visualization and editing. ACM Transactions on Graphics, 25(3), 2006

[25] J. Gray and G. J. Hancock. The propulsion of sea-urchin spermatozoa. J Exp Biol, 32(4):802-814, 1955.

[26] S. Handel and S. Imai. The free classification of analyzable and unanalyzable stimuli. Perception and Psychophysics, 12:108-116, 1972.

[27] M. Hlawitschka, G. Scheuermann, and B. Hamann. Interactive glyph placement for tensor fields. In Proc. International Symposium on Visual Computing, 2007.

[28] M. Höferlin, E. Grundy, R. Borgo, D. Weiskopf, M. Chen, I. W. Griffiths, and W. Griffiths. Video visualization for snooker skill training. Computer Graphics Forum, 29(3):1053-1062, 2010.

[29] W. V. Holt, J. O'Brien, and T. Abaigar. Applications and interpretation of computer-assisted sperm analyses and sperm sorting methods in assisted breeding and comparative research. Reproduction, Fertility and Development, 19(6):709-718, 2007.
[30] M. Horimoto, Y. Isobe, Y. Isogai, and M. Tachibana. Rat epididymal sperm motion changes induced by ethylene glycol monoethyl ether, sulfasalazine, and 2,5-hexandione. Reproductive Toxicology, 14(1):55-63, 2000

[31] E. W. Jecht and J. J. Russo. A system for the quantitative analysis of human sperm motility. Andrologia, 5(3):215-221, 1973.

[32] H. Kang, X. Chen, Y. Matsushita, and X. Tang. Space-time video montage. In Proc. IEEE Computer Vision and Pattern Recognition, volume 2, 2006.

[33] D. F. Katz and R. O. Davis. Automatic analysis of human sperm motion. Journal of Andrology, 8(3):170-181, 1987.

[34] D. F. Katz, R. O. Davis, B. A. Delandmeter, and J. W. Overstreet. Real-time analysis of sperm motion using automatic video image digitization. Computer Methods and Programs in Biomedicine, 21(3):173-182, 1985

[35] G. Kindlmann and C.-F. Westin. Diffusion tensor visualization with glyph packing. IEEE Transactions on Visualization and Computer Graphics, 12(5):1329-1335, 2006.

[36] R. M. Kirby, H. Marmanis, and D. H. Laidlaw. Visualizing multivalued data from $2 \mathrm{~d}$ incompressible flows using concepts from painting. In Proc. IEEE Visualization, pages 333-340, 1999.

[37] D. H. Krantz and A. Tversky. Similarity of rectangles: an analysis of subjective dimensions. Journal of Mathematical Psychology, 12:4 34, 1975.

[38] R. Laramee, H. Hauser, H. Doleisch, B. Vrolijk, F. Post, and D. Weiskopf. The state of the art in flow visualization: Dense and texture-based techniques. Computer Graphics Forum, 23(2):203221, 2004.

[39] R. Lavara and M. Baselga. Correlation between CASA and asma parameters in rabbit semen. Reproduction, 38(2):381-386, 2005.

[40] P. Legg, D. Chung, M. Parry, M. Jones, R. Long, I. Griffiths, and M. Chen. Matchpad: Interactive glyph-based visualization for real-time sports performance analysis. Computer Graphics Forum, 31(3):1255-1264, 2012.

[41] F. Li, A. Gupta, E. Sanocki, L. He, and Y. Rui. Browsing digital video. In Proc. ACM CHI, pages 169-176, 2000.

[42] J. Lighthill. Flagellar hydrodynamics: J. V. Neumann lecture. SIAM Rev, (18):161-230, 1976.

[43] J. A. Lucy and R. A. Shweder. Whorf and his critics: Linguistic and nonlinguistic influences on color memory. American Anthropologist, 81(3):581-615, 1979.

[44] E. Maguire, P. Rocca-Serra, S.-A. Sansone, J. Davies, and M. Chen. Taxonomy-based glyph design - with a case study on visualizing workflows of biological experiments. IEEE Transactions on Visualization and Computer Graphics, 18(12):2603-2612, 2012.

[45] S. T. Mortimer. A critical review of the physiological importance and analysis of sperm movement in mammals. Human Reproduction Update, 3(5):403-439, 1997.

[46] S. T. Mortimer. CASA-practical aspects. Journal of Andrology, 21(4):515-524, 2000.

[47] T. Munzner. A nested model for visualization design and validation. IEEE Transactions on Visualization and Computer Graphics, 15(6):921-928, 2009.

[48] U. Neisser and R. Becklen. Selective looking: Attending to visually specified events. Cognitive Psychology, 7:480-494, 1975

[49] M. Nienhaus and J. Döllner. Depicting dynamics using principles of visual art and narrations. IEEE Computer Graphics and Applications, 25(3):40-51, 2005.

[50] N. Patel and I. Sethi. Video shot detection and characterization for video databases. Pattern Recognition, 30:538-592, 1997.

[51] C. Person, T. Lester, M. Person, and R. Rorie. Computer-assisted analysis of sperm parameters after selection of motile sperm by either percoll gradient, filtration, or swim-up procedures. AAES Research Series 553.

[52] F. Post, B. Vrolijk, H. Hauser, R. Laramee, and H. Doleisch. The state of the art in flow visualisation: Feature extraction and tracking. Computer Graphics Forum, 22(4):775-792, 2003

[53] R. Ramos, G.and Balakrishnan. Fluid interaction techniques for the control and annotation of digital video. In Proc. ACM Symposium on User Interface Software and Technology, pages 105-114, 2003.

[54] T. Robeck, S. Gearhart, K. Steinman, E. Katsumata, J. Loureiro, and J. O'Brien. In vitro sperm characterization and development of a sperm cryopreservation method using directional solidification in the killer whale (orcinus orca). Theriogenology, 76(2):267-279, 2011 
[55] T. Ropinski, S. Oeltze, and B. Preim. Survey of glyph-based visualization techniques for spatial multivariate medical data. Computers \& Graphics, 2011.

[56] T. Ropinski and B. Preim. Taxonomy and usage guidelines for glyphbased medical visualization. In Proc. Conference on Simulation and Visualization, pages 121-138, 2008.

[57] T. Ropinski, M. Specht, J. Meyer-spradow, K. Hinrichs, and B. Preim. Surface glyphs for visualizing multimodal volume data. In Proc. Workshop on Vision, Modeling, and Visualization, pages 3-12, 2007.

[58] M. Rubinstein, A. Shamir, and S. Avidan. Improved seam carving for video retargeting. ACM Transactions on Graphics, 27(3):1-9, 2008

[59] K. Schoeffmann and L. Boeszoermeny. Video browsing using interactive navigation summaries. In Proc. International Workshop on Content-Based Multimedia Indexing, pages 243-248, 2009.

[60] R. N. Shepard. Attention and metric structure of the stimulus space. Journal of Verbal Learning and Verbal Behavior, (1):54-87, 1964.

[61] D. J. Smith, E. A. Gaffney, H. Gadêlha, N. Kapur, and J. C. KirkmanBrown. Bend propagation in the flagella of migrating human sperm, and its modulation by viscosity. Cell Motility and the Cytoskeleton, 66(4):220-236, 2009.

[62] P. Steigerwald and W. Krause. Estimation of sperm morphology using a new CASA system. Andrologia, 30(1):23-27, 1998.

[63] S. Stokes. Visual literacy in teaching and learning: A literature perspective. Electronic Journal for the Integration of Technology in Education, 1(1):10-19, 2002.

[64] C. Tretipskul, K. Buranaamnuay, S. Koonjaenak, P. Tumaruk, and M. Techkumphu. The use of computer-assisted sperm analysis for discriminating series of motility pattern of frozen-thawed boar semen. Thai Journal of Veterinary Medicine, 40(1):25-30, 2010.

[65] V. S. Truong B. Video abstraction: A systematic review and classification. ACM Transactions on Multimedia Computing, Communications and Applications, 3(1), 2007.

[66] M. Ward. Multivariate data glyphs: Principles and practice. In Handbook of Data Visualization, pages 179-198. 2008.

[67] M. O. Ward. A taxonomy of glyph placement strategies for multidimensional data visualization. Information Visualization, 1(3/4):194210, 2002.

[68] C. Ware. Visual Thinking: for Design. Morgan Kaufmann, 2008.

[69] P. C. Wong, H. Foote, D. L. Kao, R. Leung, and J. Thomas. Multivariate visualization with data fusion. Information Visualization, 1(3/4):182-193, 2002

[70] World Health Organization. WHO laboratory manual for the Examination and processing of human semen. World Health Organization, 5th edition, 2010. Department of Reproductive Health and Research.

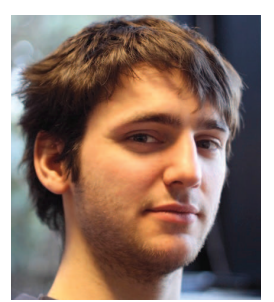

Brian Duffy received a BSc in computer science from University College Dublin, Ireland in 2006 . He received his $\mathrm{PhD}$ in visualization and computer graphics from the the same institution in 2011. He is currently working for the Oxford Centre for Computational and Applied Mathematics at the University of Oxford. His research interests include scientific visualization with application to mathematical problems.

Jeyarajan Thiyagalingam received his $\mathrm{PhD}$ degree in computer science from Imperial College, London, in 2005. At present, he is a James Martin Fellow at the University of Oxford. His research interests include high performance computing, parallel algorithms, efficient algorithms for image and video processing, target tracking, estimation and data processing.

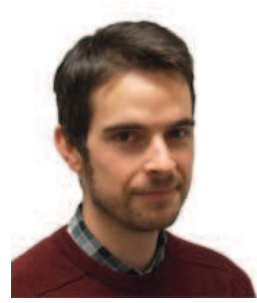

Simon Walton completed his $\mathrm{PhD}$ in 2007 at Swansea University, focusing on the deformation of discretely sampled object representations using GPUs. He now works as a research associate at the Oxford e-Research Centre, Oxford University. His recent interests are in Video Visualization techniques with a focus on perceptual challenges of combining many different layers of information in the same scene.

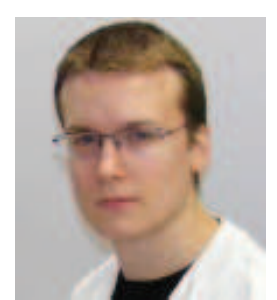

David J. Smith is in Applied Mathematics at the University of Birmingham. He received his PhD from the same institution in 2006 and subsequently undertook multidisciplinary training in the Schools of Medicine and Mathematics, funded by MRC and Wellcome Trust, specialising in the biofluiddynamics of sperm and cilia. He was appointed Birmingham Science City Fellow and Lecturer in 2009

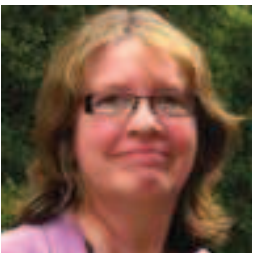

Anne Trefethen is Chief Information Officer, Professor of Scientific Computing and Associate Director of the e-Research Centre at Oxford University. Her career has spanned both industry and academia, including ten years in the USA, where she worked for Thinking Machines Corp and at the Theory Centre, Cornell University. Before coming to Oxford, Anne was a Director of the UK eScience Core Programme and was VP for Research and Development at NAG Ltd. Anne's work has focused on high-performance computing and data intensive science and particularly on algorithms and software systems.

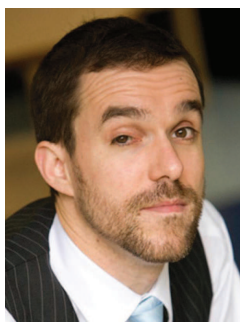

Jackson C. Kirkman-Brown received his $\mathrm{PhD}$ in reproductive science from the University of Birmingham in 2000. He has extensive experience in the study of signalling in sperm for human fertility. He is currently the lead scientist for Assisted Conception (IVF) services. His research interests are on improving diagnosis and refining rational treatments for infertility, alongside developing research in all aspects of patient treatment and care through multi-disciplinary collaboration. Currently his team of cell biologists are working with industrial and academic partners across a wide range of disciplines, such as mathematics, computer science, optical engineering, rheology, fluid mechanics and chemistry, examining human sperm motility and modulation.

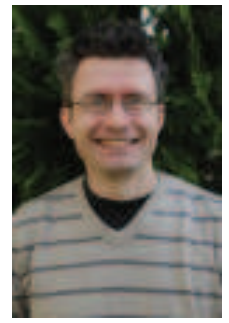

Eamonn A. Gaffney MA PhD (Cantab). University Lecturer, Centre for Mathematical $\mathrm{Bi}$ ology, Mathematical Institute, University of Oxford. Research interests: biological and biomedical applications of mathematics including flagellated swimming mechanics, biological aspects of reaction diffusion systems ranging from self-organisation models to oxygen transport in tissue and models of cell movement, signalling and interaction.

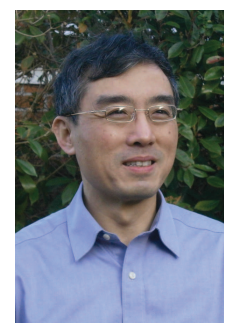

Min Chen received the $\mathrm{PhD}$ degree from University of Wales in 1991. He is currently a professor of scientific visualization at Oxford University and a fellow of Pembroke College. Before joining Oxford, he held research and faculty positions at Swansea University (i.e., research officer from 1987, lecturer from 1990, senior lecturer from 1998 and professor from 2001). His research interests include visualization, computer graphics and human-computer interaction. His services to the research community include papers co-chair of IEEE Visualization 2007 and 2008, co-chair of Volume Graphics 1999 and 2006, member of many program committees and several editorial boards, and co-director of Wales Research Institute of Visual Computing. He is a fellow of BCS, EG and LSW. 